\title{
Looking into a Better Future: Novel Therapies for Metastatic Melanoma
}

\author{
Alessia Villani (D) - Massimiliano Scalvenzi · Gabriella Fabbrocini • \\ Jorge Ocampo-Candiani · Sonia Sofía Ocampo-Garza
}

Received: March 2, 2021 / Published online: April 17, 2021

(C) The Author(s) 2021

\begin{abstract}
Even though melanoma represents a small percentage of all cutaneous cancers, it is responsible for most deaths from skin neoplasms. In early stages it can be successfully treated with surgery, but as the disease expands the survival rate drops significantly. For many years the mainstay of treatment for metastatic melanoma was chemotherapeutic agents, even though they failed to prove survival prolongation. After the advent of ipilimumab, a survival benefit and better overall response rate could be offered to the patients. Other new therapies, such as immunotherapies, targeted therapies, vaccines, and small molecules, are currently being studied. Also, combination regimens have demonstrated superiority to some monotherapies. Nowadays, ipilimumab should no longer be considered the first-line therapy given its severe
\end{abstract}

Alessia Villani and Massimiliano Scalvenzi are contributed equally to the manuscript.

A. Villani $(\bowtie) \cdot$ M. Scalvenzi · G. Fabbrocini ·

S. S. Ocampo-Garza

Dermatology Unit, Department of Clinical Medicine

and Surgery, University of Naples Federico II,

Naples, Italy

e-mail: ali.vil@hotmail.it

J. Ocampo-Candiani · S. S. Ocampo-Garza

Dermatology Department, Universidad Autónoma

de Nuevo León, University Hospital “Dr. José

Eleuterio González", Monterrey, NL, Mexico toxicity and lower efficacy, while nivolumab remains efficacious and has a good safety profile. T-VEC as monotherapy has been shown to be an elegant alternative even for the elderly or cases of head and neck melanomas. If the BRAF mutation status is positive, the combination of dabrafenib and trametinib could be an option to consider. Despite the success of the novel treatments, their effectiveness is still limited. New studies have opened up new avenues for future research in melanoma treatment, which is expected to lead to better therapeutic outcomes for our patients. The objective of this review is to discuss the novel therapies for metastatic melanoma that have been tested in humans during the last 3 years to obtain a sharper perspective of the available treatment options for specific patient characteristics.

Keywords: Melanoma; Metastatic melanoma; Targeted therapy; Immune checkpoint inhibitors; Vaccines; Small molecules 


\section{Key Summary Points}

Even though melanoma represents a small percentage of all cutaneous cancers, it is responsible for most deaths from skin cancers

The survival rate at 5 years for localized melanoma is $98.3 \%$ while for metastatic melanoma (MM) is $16 \%$

In the last decade melanoma clinical research has completely changed the scenario of therapeutic approaches for patients with unresectable advanced melanoma

Multiple targets for drug development have been identified to treat advanced melanoma and are currently undergoing development

\section{DIGITAL FEATURES}

This article is published with digital features, including a summary slide, to facilitate understanding of the article. To view digital features for this article go to https://doi.org/10.6084/ m9.figshare.14317787.

\section{INTRODUCTION}

Cutaneous melanoma is responsible for most deaths from cutaneous neoplasms with an increasing incidence worldwide [1, 2]. Survival rates vary depending on tumor stage at the time of diagnosis, which depends on the depth of the tumor (Breslow) as well as lymph node involvement or distant metastasis [3]. Stage I and II involve localized disease, stage III is characterized by metastasis to the local lymph nodes, and stage IV represents distant metastasis [4].

The survival rate at 5 years for localized melanoma is $98.3 \%$ while for metastatic melanoma (MM) is $16 \%$ [3]. Surgery remains the first treatment option for resectable melanoma [2]. For many years the mainstay of treatment for MM was chemotherapeutic agents, even though they failed to prove survival prolongation $[3,5]$. Finally, in 2010 the advent of ipilimumab changed the overall response rate (ORR) and offered a survival benefit [5]. After ipilimumab, other new therapies, such as immunotherapies, targeted therapies, vaccines, small molecules, and combination therapies, have changed the prognosis for patients with MM. Although nowadays patients can be offered a wider variety of therapies, many characteristics have to be taken into account, such as the presence of melanoma genome mutations, specific features of the tumor, comorbidities and tolerability of the patient, as well as risks associated with treatment [2].

In this study we discuss the novel therapies for metastatic melanoma that have been tested in humans during the last 3 years to obtain a sharper perspective related to the available treatment options for specific patient characteristics.

\section{METHODS}

A literature search on PubMed, Medline, EBSCO, Google Scholar, and the Cochrane Library databases regarding treatment of metastatic melanoma from January 2018 to February 2021 was made. Reviews, metanalyses, clinical trials (CT), real-life studies (RLS), case reports, and series were reviewed. The most relevant articles were included. A revision of the references was also made to include articles that could have been missed. Assessment of treatment efficacy was made through overall survival (OS), progression-free survival (PFS), recurrence-free survival (RFS), disease-free survival (DFS), durable response rate (DRR), and overall response rate (ORR). A summary of available therapies for $\mathrm{MM}$ is shown in Fig. 1. All included studies are shown in Table 1. This article is based on previously conducted studies and does not contain any studies with human participants or animals performed by any of the authors. 


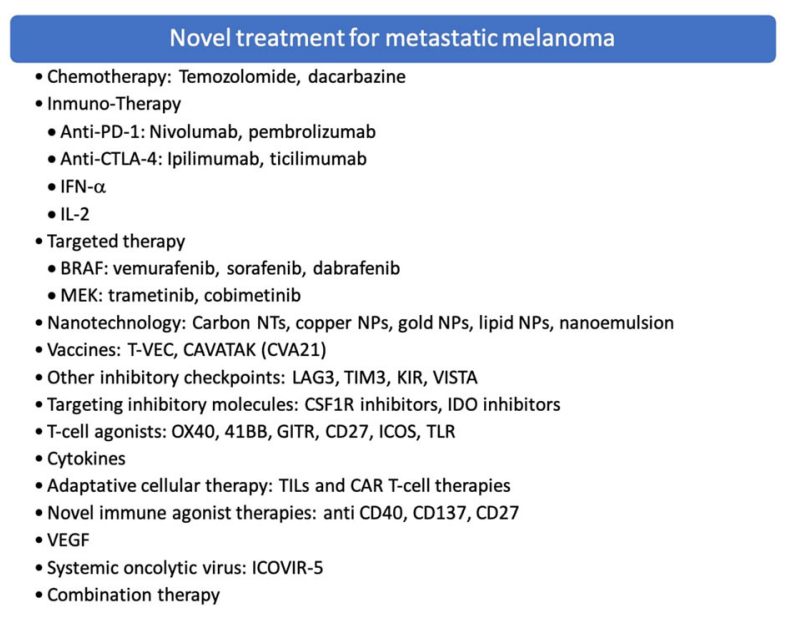

Fig. 1 Summary of some of the available therapies for metastatic melanoma

\section{Immune Checkpoint Inhibitors}

Programmed Cell Death protein-1 (PD-1) and cytotoxic T-Lymphocyte-associated antigen 4 (CTLA-4) are immune checkpoint molecules that downregulate $\mathrm{T}$ cell activation pathways, important in the immune tolerance. PD-1 is expressed on activated T cells, B and NK cells, and monocytes. It binds to its ligand PDL-1 and inhibits the signaling of $\mathrm{T}$ cell receptor, preventing $\mathrm{T}$ cell activation and the release of proinflammatory cytokines. CTLA- 4 is a homo$\log$ of CD28 and is expressed on regulatory $\mathrm{T}$ cells, inhibiting signals to T cells [6].

\section{Anti-PD1}

In 2020, a retrospective, single-center analysis with stage III/IV melanoma patients treated with single-agent anti-PD1 was conducted [7]. Three hundred ninety-six patients with at least 3 months of follow-up after discontinuation of therapy were included in the study. Most of the subjects were treated with pembrolizumab $(85.9 \%)$ and the rest with nivolumab (14.1\%). The median follow-up in patients that survived was 28.9 months, and the median time of treatment was 4.8 months. The most common reasons for treatment discontinuation were disease progression (DP) (49.5\%) and toxicity $(21.7 \%)$. At 5 years after the start of treatment, the treatment failure-free survival was $21.5 \%$.
Five-year OS was $40.9 \%$, and the median OS was 39 months. In $25.8 \%$ of the included patients, the oncologist considered a complete response (CR). At 3 years the probability of being alive and of not requiring other therapy was $72.1 \%$. Patients with M1b disease were more likely to have CR compared to other stage IV and stage III combined [7].

Nivolumab In CheckMate 037, a randomized, controlled, open-label phase 3 trial, patients with advanced melanoma (stage IIIC or IV disease) were randomized to receive either intravenous (IV) nivolumab $3 \mathrm{mg} / \mathrm{kg}$ every 2 weeks or the investigator's choice of chemotherapy (ICC) (dacarbazine $1000 \mathrm{mg} / \mathrm{m}^{2}$ every 3 weeks or carboplatin area under the curve 6 plus paclitaxel $175 \mathrm{mg} / \mathrm{m}^{2}$ every 3 weeks) [8]. Patients were treated until progression or unacceptable toxicity, with follow-up of 2 years. In total, 272 patients were assigned to nivolumab and 133 to ICC. More patients treated with nivolumab had brain metastases or increased lactate dehydrogenase levels at baseline. Median OS for nivolumab patients was 15.7 months, compared to 14.4 months in the ICC group. Median PFS was 3.1 months and 3.7 months in the nivolumab and ICC group, respectively. Overall response rate was higher in the nivolumab group (27\% versus $10 \%)$. Treatment-related AEs were seen in $77 \%$ and $82 \%$ of patients in the nivolumab and ICC groups. Nivolumab demonstrated a more durable response, but with no difference in survival compared to the ICC [8].

A double-blind, randomized, controlled phase 3 adjuvant trial for patients with resected stage IIIB, IIIC, or IV melanoma was conducted in 2017 (CheckMate 238) [9]. This study provided significant improvement in recurrencefree survival as well as in distant metastasis-free survival in patients treated with nivolumab versus ipilimumab. Later, Ascierto et al. [10] published an updated 4-year report of efficacy, overall survival, and late-emergent safety.

Eligible patients were randomly assigned to receive either nivolumab $3 \mathrm{mg} / \mathrm{kg}$ IV every 2 weeks or ipilimumab $10 \mathrm{mg} / \mathrm{kg}$ IV every 3 weeks for 4 doses and then every 12 weeks, for up to 1 years or until DR, toxicity, or withdrawal 


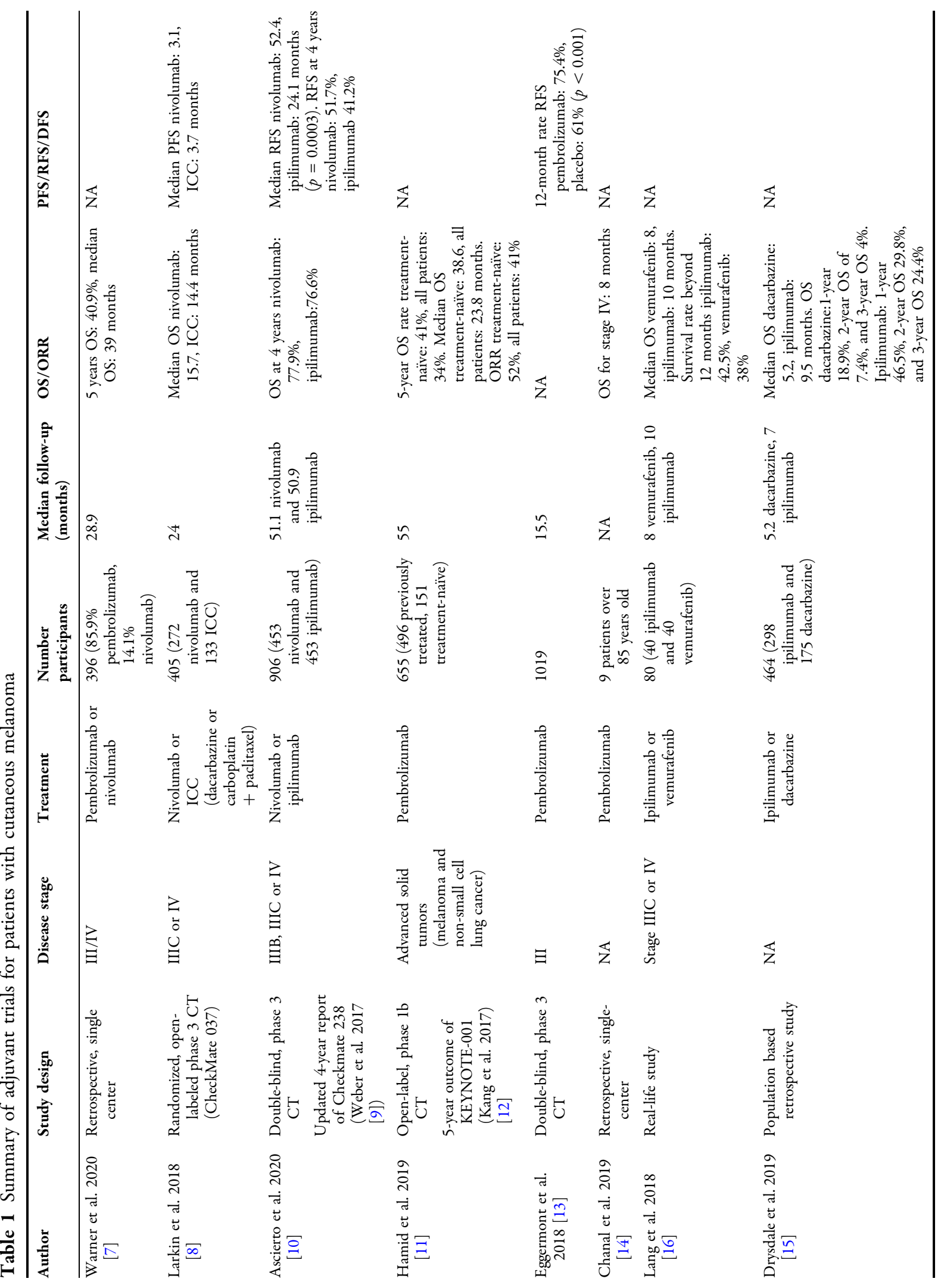




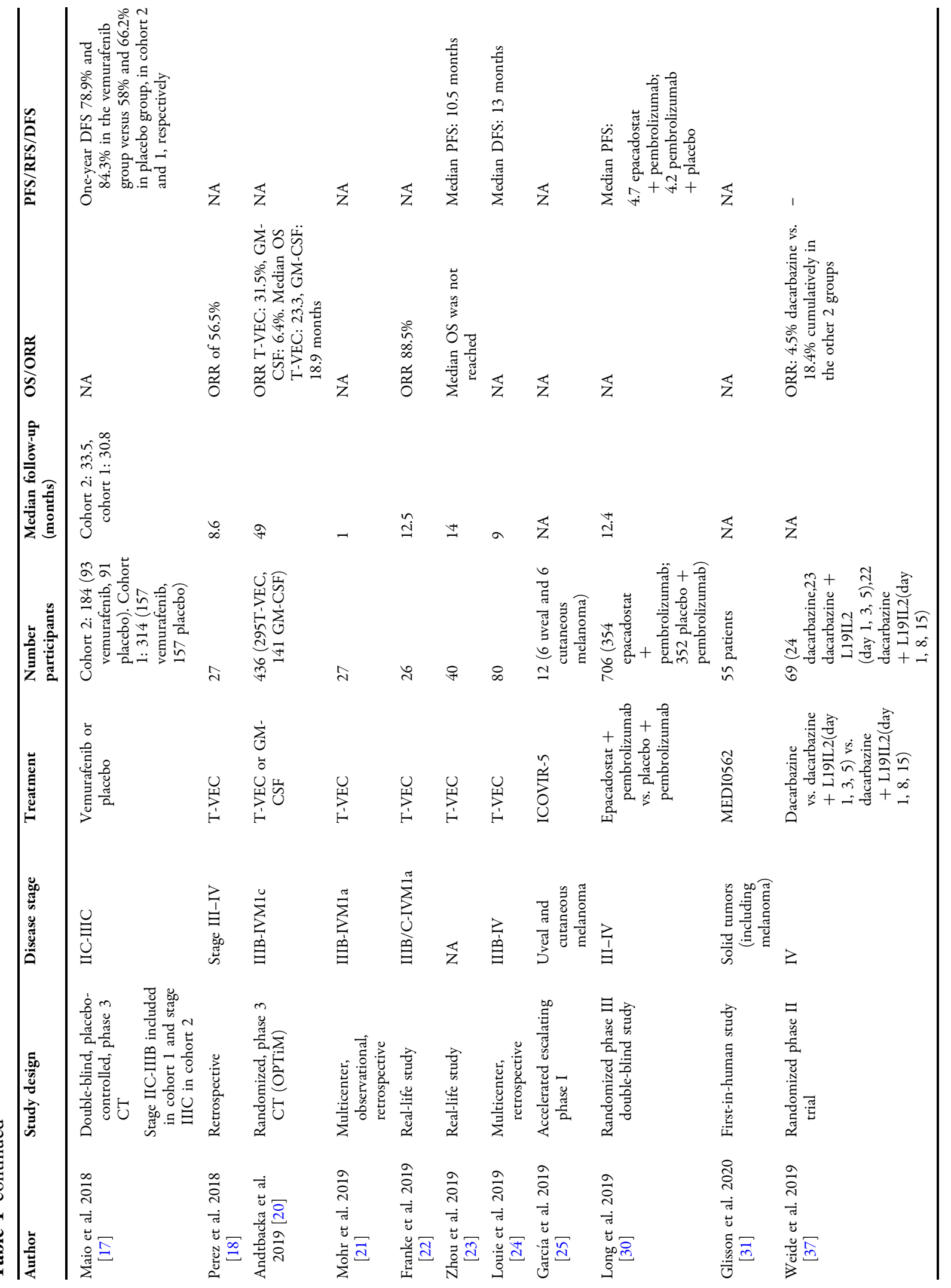




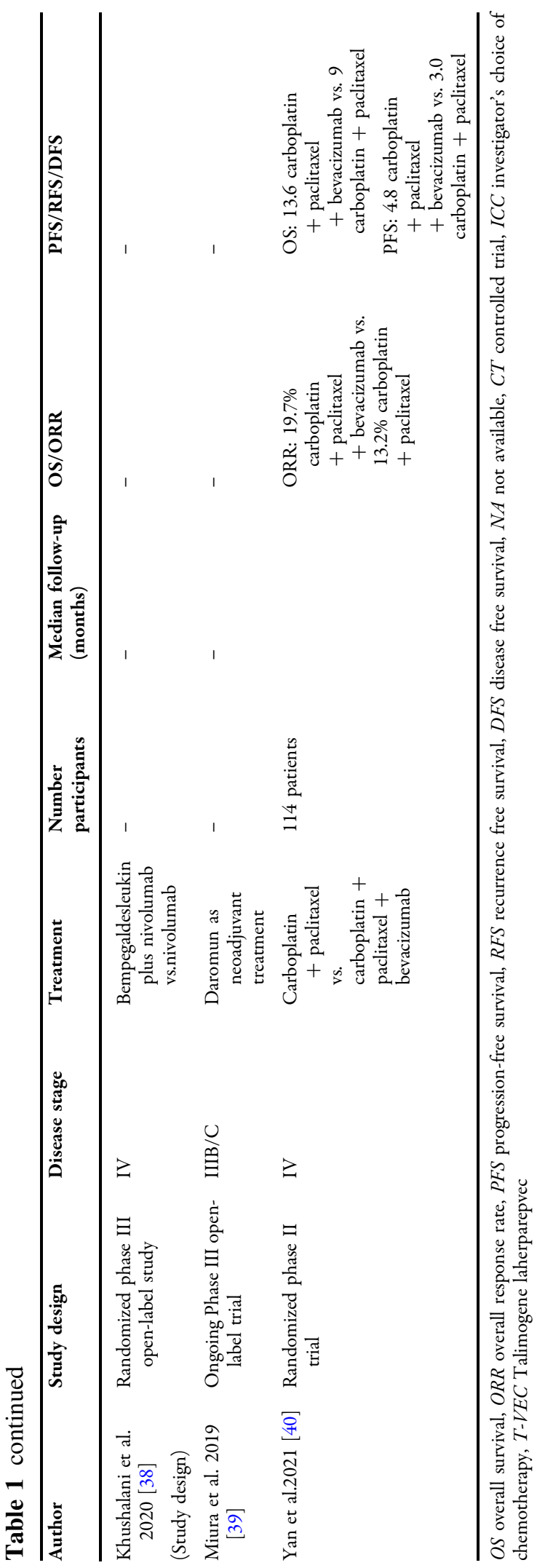

of consent [10]. In total, 906 patients were randomized to nivolumab (453) or ipilimumab (453). Median follow-up was 51.1 months for nivolumab and 50.9 months for ipilimumab. All patients had completed or discontinued treatment by the 18-month analysis. After the database lock 316 patients in the nivolumab group and 298 patients in the ipilimumab group continued in the study. At a minimum of 4 years of follow-up, 212 recurrence events were found in the nivolumab group and 253 in the ipilimumab group. Median RFS in the patients treated with nivolumab was 52.4 months and 24.1 months for the ipilimumab group $(p=0.0003)$. RFS at 4 years was $51.7 \%$ and $41.2 \%$ in the nivolumab and ipilimumab group, respectively, and an absolute risk difference of $10.5 \%$ was found. The OS at 4 years was $77.9 \%$ and $76.6 \%$ with nivolumab and ipilimumab. Late-emergent treatment-related adverse effects (AEs) were found in $4 \%$ of the nivolumab and $6 \%$ of the ipilimumab group, while grade $3-4$ AEs were reported in $1 \%$ of the nivolumab and $2 \%$ of the ipilimumab group. No patients in the nivolumab group died because of toxicity, while two patients in the ipilimumab group died because of marrow aplasia. Nivolumab seems to have a more tolerable safety profile than ipilimumab, with good efficacy [10].

Pembrolizumab In 2019 Hamid et al. [11] reported the 5-year outcomes of the KEYNOTE001 study [12], an open-label, phase $1 \mathrm{~b}$ CT that included patients with advanced solid tumors, such as melanoma and non-small cell lung cancer [12]. Six hundred fifty-five patients with a diagnosis of advanced or metastatic melanoma were included; 496 were previously treated and 151 were treatment naïve. They received pembrolizumab $2 \mathrm{mg} / \mathrm{kg}$ every 3 weeks, $10 \mathrm{mg} / \mathrm{kg}$ every 3 weeks, or $10 \mathrm{mg} / \mathrm{kg}$ every 2 weeks until progression of disease, toxicity, or patient or investigator decision to retract. Median follow-up was 55 months and median duration of exposure to pembrolizumab was 5.6 months ( 1 day to 67 months). At time of data cut-off, 33 patients $(5 \%)$ were still on treatment; 569 patients $(87 \%)$ discontinued treatment, 275 (42\%) because of DP, 166 (25\%) adverse events, 80 (12\%) physician choice, 36 
(5\%) patient withdrawal, and $8(1 \%)$ violation of protocol, and 4 were lost to follow-up.

At data cutoff, 81 patients $(54 \%)$ of treatment-naïve patients and $412(63 \%)$ of all patients had died. The 5 -year OS rate was $41 \%$ in treatment-naïve patients and $34 \%$ in all patients. Median OS was 38.6 months in treatment-naïve and 23.8 months in all patients. Among all treated patients $(n=655), 104(16 \%)$ achieved CR, and 163 (25\%) partial response (PR); 156 (24\%) had stable disease (SD), and the disease control rate (DCR) was 65\%. Median time to response was $2.8(0.5-49.6)$ months. AEs related to treatment occurred in 562 patients (86\%). Sixty-five (10\%) discontinued therapy because of treatment-related AEs, 114 (17\%) experienced grade 3-4 AEs, and none had treatment-related death. This study represents the longest pembrolizumab follow-up in patients with MM [11].

A phase 3 double-blind trial evaluated pembrolizumab as adjuvant treatment in patients with high-risk stage III melanoma [13]. A total of 1019 patients were randomized to receive either pembrolizumab $200 \mathrm{mg}$ IV $(n=514)$ or placebo $(n=505)$ every 3 weeks for a total of 18 doses (approximately 1 year). Of the patients treated with pembrolizumab, $13.8 \%$ (70) discontinued treatment for AEs and of the placebo group 13\% (66). As for discontinuation due to disease recurrence, 109 patients (21.4\%) in the pembrolizumab and 179 (35.7\%) in the placebo group discontinued the regimen. Overall median duration of follow-up was 15.1 months. The 12 -month rate of RFS was $75.4 \%$ and $61 \%$ in the pembrolizumab and placebo group, respectively. A significant difference was found in the RFS between pembrolizumab and placebo $(p<0.001)$. In total, 351 patients had a first recurrence of disease or died: 135 for the pembrolizumab and 216 for the placebo group. AEs occurred in 396 patients $(77.8 \%)$ in the pembrolizumab and 332 patients (66.1\%) in the placebo group. AEs of grade 3-5 occurred in $14.7 \%$ vs. $3.4 \%$ in the pembrolizumab and placebo group. One pembrolizumab-related death due to myositis occurred [13].

A monocentric retrospective case series included nine patients with MM over 85 years old [14]. All patients were treated with pembrolizumab IV ( $2 \mathrm{mg} / \mathrm{kg}$ every 3 weeks). One patient had been previously treated with dacarbazine; the rest received pembrolizumab as first-line treatment. Mean number of infusions was 4; however, the five patients with stage IV disease were able to receive only three or fewer infusions. Seven patients had treatment-related AEs of grade 3 or 4 . One patient died suddenly at home 4 days after infusion so a fatal AE could not be ruled out. The results in patients that received at least two infusions (6 patients) were one CR, two PRs, and 3 DPs. Two patients received just one infusion; one died 1.5 months from DP, and the other had partial regression. At the end of the study, 8 patients had died. The OS was 8 months for stage IV patients. Pembrolizumab treatment in patients older than 85 years, even when a reduced number of infusions are delivered, may induce a response, but it may also be associated with a higher risk of toxicity [14].

\section{Anti-CTLA-4}

Ipilimumab Ipilimumab is an anti-CTLA-4 monoclonal antibody; it was the first checkpoint inhibitor for treating metastatic and unresectable melanoma that showed efficacy [15]. Lang et al. [16] published a real-life study in which they included 40 patients treated with vemurafenib $960 \mathrm{mg}$ twice daily (BID) and 40 patients treated with ipilimumab IV $3 \mathrm{mg} / \mathrm{kg}$ body weight every 3 weeks for four infusions. Patients in the vemurafenib group had BRAF $^{\mathrm{V} 600 \mathrm{E}}$ mutation, while $87.5 \%$ of the ipilimumab group were wild type. Both groups presented with metastasis in the lymph nodes, lung, liver, and skin; $90 \%$ of the patients were in stage M1c before beginning the therapy. Vemurafenib was applied as first-line therapy in most of the patients (85\%), while ipilimumab was second line in most patients (85\%). Mean duration of treatment for vemurafenib was 11.4 months; $32.5 \%$ required a reduction of the dose, and $12.5 \%$ had an early termination due to adverse events (AEs). Of the patients treated with ipilimumab $47.5 \%$ received the four infusions; $32.5 \%$ had a reduced number of infusions due to side effects and $20 \%$ due to massive tumor progression. Median OS in the vemurafenib group was 8 months $( \pm 1.25 ; 95 \%$ CI: 
5.55-10.45) and 10 months $( \pm 3.16 ; 95 \% \mathrm{CI}$ : 3.81-16.19) in the ipilimumab group. No significant difference between groups was found $(p=0.689)$. Survival rate beyond 12 months was $42.5 \%$ in the ipilimumab group and $38 \%$ in the vemurafenib group, and after 24 months 35.5 and $25.5 \%$, respectively. Among the patients treated with vemurafenib, the survival without brain metastasis was significantly longer compared to those with brain metastasis $(\log$-rank $p$ value $=0.013) . \quad$ Long-term survival ( $\geq 24$ months) was reached by $32.5 \%$ of patients $(57.7 \%$ ipilimumab and $42.3 \%$ vemurafenib) [16].

A population-based study of patients treated for metastatic or unresectable melanoma with ipilimumab or dacarbazine was published in 2019 [15]. The control group included 175 patients treated with dacarbazine and 289 patients treated with ipilimumab. Among the control group, 41 patients received dacarbazine combined with a platinum agent and/or cytotoxic agents. Median follow-up was 5.2 months for dacarbazine and 7 months for the ipilimumab group. Median survival was 5.2 months for dacarbazine and 9.5 months for ipilimumab. One-year OS was $18.9 \%$, 2-year OS $7.4 \%$, and 3 -year OS $4 \%$ for dacarbazine, while for ipilimumab they were $46.5 \%, 29.8 \%$, and $24.4 \%$, respectively. The improved OS was greater in patients treated with ipilimumab who were younger than 65 years old, male sex, and with no comorbidities [15].

\section{Targeted Therapy}

\section{BRAF}

Approximately half of cutaneous melanomas have an activating mutation in the BRAF oncogene, which leads to a constitutive activation of the mitogen-activated protein kinase (MAPK) signaling pathway. Several drugs that target BRAF mutant cells have been proposed as treatment for metastatic melanomas, including vemurafenib and dabrafenib [1].

Vemurafenib Maio et al. [17] performed a phase 3, double-blind, randomized, placebocontrolled study with $\mathrm{BRAF}^{\mathrm{V} 600}$ mutation- positive melanoma stage IIC to IIIC patients (BRIM8). Subjects were randomly assigned to receive either vemurafenib $960 \mathrm{mg}$ BID or matching placebo for 52 weeks $(13 \times 28$-day cycles). Patients with stage IIC-IIIB were included in cohort 1 and patients with stage IIIC in cohort 2. In total, 184 patients (93 vemurafenib and 91 placebo) were enrolled in cohort 2 and 314 patients (157 vemurafenib and 157 placebo) in cohort 1 . At the time of data cutoff, median follow-up was 33.5 months in cohort 2 and 30.8 months in cohort 1 . In cohort $2,30 \%$ of the vemurafenib group and $34 \%$ of the placebo discontinued the study. In cohort $1,23 \%$ of the vemurafenib group and $26 \%$ of the placebo withdrew [17]. In cohort 2, median disease-free survival (DFS) was 23.1 months in the vemurafenib group and 15.4 months in the placebo group. In cohort 1 , median DFS was not reached in the vemurafenib group. One-year DFS was $78.9 \%$ and $84.3 \%$ in the vemurafenib group versus $58 \%$ and $66.2 \%$ in the placebo group in cohort 2 and 1, respectively. At data cutoff, 83 deaths had occurred in the overall population. Thirty-five deaths occurred in the vemurafenib group (16 in cohort 1 and 19 in cohort 2) and 47 in the placebo group (28 in cohort 1 and 19 in cohort 2). Regarding the safety profile, the most frequently reported AEs were skin-related events (rash and alopecia), arthralgia, nausea, and fatigue. Grade 3 or 4 events were found in 141 patients (57\%) and 37 (15\%) of the vemurafenib and control group, respectively. Serious AEs occurred in 40 patients (16\%) and 25 patients (10\%) of the vemurafenib and control group, the most common being basal cell carcinoma. One fatal event, which was not considered drug related, was reported in the vemurafenib group [17].

\section{Vaccines}

\section{Talimogene Laherparepvec (T-VEC)}

Talimogene laherparepvec (T-VEC) is a modified herpes simplex virus 1 (HSV-1). It recruits antigen-presenting cells and expresses granulocyte macrophage-colony-stimulating factor (GMCSF) [18]. This virus infects and replicates inside tumor cells, causing lysis of the infected 
tumoral cells. T-VEC is administered intralesionally in cutaneous, subcutaneous, and nodal lesions in stage IIIB, IIIC, and IVM1a melanomas after initial surgery [19].

A retrospective study including 27 patients with melanoma, $81.5 \%$ with stage III disease, and $18.5 \%$ with stage IV was made in 2018 [18]. Thirteen patients were previously treated with regional or systemic therapies. Patients were injected with an initial dose of $10^{6}$ plaqueforming units (PFU) per milliliter; 3 weeks later they received a second injection at a dose of $10^{8}$ PFU per milliliter, which was continuously repeated every 2 weeks until DP, CR, or intolerance to therapy. Median follow-up was 8.6 months $(2.8-20.5)$. Three patients did not complete the 8-week follow-up and were excluded from analysis; $43.5 \%$ experienced CR and $13 \%$ PR, and $21.7 \%$ had stable disease (SD). An overall response rate of $56.5 \%$ was found and a disease control rate of $78.3 \%$. Five patients presented DP. Median time to response was 2.1 months. A significant association between disease stage and treatment response was found $(p=0.041)$, with $100 \%, 39 \%$, and $50 \%$ of patients responding to treatment with stage IIIB, IIIC, and IV, respectively. Most AEs were limited to mild constitutional symptoms within $48 \mathrm{~h}$ of injection [18].

In OPTiM, a randomized phase III CT for patients with unresectable stage IIIB-IVM1c melanoma, participants were randomized 2:1 to receive intratumoral T-VEC or subcutaneous recombinant GM-CSF [20]. Of the 436 included patients, 295 were treated with T-VEC and 141 with GM-CSF. Treatment was given for 6 months. After this time, therapy was continued until DP, consent withdrawal, intolerability, lack of response, CR, or lack of response by 12 months. Median duration of treatment was 10 weeks in the GM-CSF and 23.1 weeks in the T-VEC group. Median follow-up was 49 months. DRR was higher in the population treated with T-VEC vs. GM-CSF (19.3\% vs. $1.4 \%)$. ORR was also higher in the T-VEC arm (31.5\% vs. $6.4 \%)$. Fifty $(16.9 \%)$ and 1 patient in the T-VEC and GM-CSF group, respectively, achieved a CR; 43 $(14.6 \%)$ and $8(5.7 \%)$ achieved a PR. Median time to CR in the arm treated with T-VEC was 8.6 months. CR achievement with T-VEC was significantly associated with a baseline tumor burden of $<14.5 \mathrm{~cm}^{2}$ and an early melanoma stage. Median OS with T-VEC was 23.3 months and 18.9 months with GM-CSF. Most common AEs were chills, pyrexia, fatigue, influenza-like symptoms, and nausea. Grade 3/4 treatmentrelated AEs were found in 33 patients (11.3\%) in the T-VEC patients. The only AE that occurred in more than two patients was cellulitis [20].

A multicenter, observational, retrospective review that included patients with stage IIIBIVM1a melanoma treated with T-VEC was made in Germany [21]. Twenty-seven patients were included; $56 \%$ had stage IIIB/C and the remaining 44\% stage IVM1a. All the included patients had a history of prior surgery, and more than half had a sentinel lymph node biopsy (SLNB) performed. Also, about half of the patients received a prior local treatment before T-VEC (radiation, electrochemotherapy, IL-2 or interferon-alfa, or local ablation). At the end of the study, 13 patients had ongoing and 14 had discontinued therapy. Three patients had no remaining injectable lesions and seven $\mathrm{PD}$; three discontinued because of patients' decisions and and one because of side effects (nausea). The median duration of treatment was 22.1 weeks, and the median duration of followup was 30.6 weeks [21]. Another real-life study in patients with stage IIIB/C-IVM1a melanoma treated with T-VEC was made by Franke et al. [22]. Twenty-six patients were included, with a median follow-up time of 12.5 months. Best ORR was $88.5 \%$, CR was $61.5 \%$, and DCR $92.3 \%$. Eight patients $(30.8 \%)$ had PD. Two patients were re-treated with T-VEC because of locoregional recurrence after a prior CR; both reached a CR again. AEs occurred in $100 \%$ of patients, but serious AEs only occurred in one patient (pancolitis). This study had a higher ORR than most clinical trials, which could be explained by the fact that no stage IV patients were treated [22]. Later, a real-life study by Zhou et at. [23] that included 40 patients with MM treated with T-VEC was made. Of the included patients, $85 \%$ had $\leq$ stage IV M1a and $15 \%$ had stage $\geq$ IV M1b-d; $47.5 \%$ had any tumor $\geq 2 \mathrm{~cm}$. Eighty percent of the patients had received prior therapies (35\% conventional or targeted therapy and $45 \%$ immune checkpoint inhibitors). As 
results, 17 patients (42.5\%) had CR and $2(5 \%)$ $\mathrm{PR}$, and the remaining patients had either DP or were non-responders. Median follow-up was 14 months (1.9-26.7 months). Median PFS was 10.5 months. Patients with poorer performance status defined by the Eastern Cooperative Oncology Group (ECOG) had significantly decreased OS $(p=0.0049)$ and PFS $(p=0.03)$. Also, larger tumors $(\geq 2 \mathrm{~cm})$ had significantly decreased OS $(p=0.046)$, PFS $(p=0.0085)$, and DRR $(p=0.029)$. Age $\geq 75$ years did not affect OS compared to patients aged $<75$ years. AEs were mild (fevers and fatigue), with three patients $(7.5 \%)$ discontinuing treatment because of AEs. No treatment-related deaths were seen [23]. The largest published series on the efficacy and safety of T-VEC in locoregional advanced melanoma patients included 80 patients with a median follow-up time of 9 months [24]. Twenty-one patients (36\%) had head and neck as the primary site of disease. Most patients underwent systemic or regional therapy before receiving T-VEC (57.5\%). Six patients did not have a previous resection. Thirty-six patients (46\%) had clinical stage IIIB disease, 25 had stage IIIC (31\%), 1 had clinical stage IIID, and $16(20 \%)$ had distant metastasis at the time of treatment. The included patients received a median of five cycles of T-VEC. Twenty-nine patients (37\%) had CR, 6 (8\%) PR, 15 patients (19\%) SD, and 25 (30\%) DP. Fifty-eight percent of patients did not have any $\mathrm{AE}, 28 \%$ had constitutional, flu-like symptoms, and $6 \%$ had an early termination of treatment because of AEs ( 1 cold sore, 4 infections). Patients with stage IIIB were more likely to have CR (68\%) compared to stage IIIC, IIID, and IV (26\%, 0\%, and 6\% respectively). As the authors showed, T-VEC can be used in locally advanced head and neck melanomas with a good response [24].

\section{Intravenous Oncolytic Virus}

\section{ICOVIR-5}

The first-in-human phase I trial of IV administration of ICOVIR-5 that included patients with uveal and cutaneous metastatic malignant melanoma was conducted by García et al. [25].
ICOVIR- 5 is derived from an adenovirus type 5 that is responsive to the pRB pathway, a pathway that is deregulated in many tumors. This was an accelerated escalating phase I design with a cohort of 1 patient in case no more than grade 2 toxicity was found and later with a cohort of 3-6 patients after the first grade 3 toxicity was found. A dose-limiting-toxicity (DLT) observation period of 4 weeks was established before starting the next dose level for the first patient [25].

Thirteen patients were included in the study: six had uveal and six cutaneous melanomas. Six patients $(50 \%)$ had received at least one regimen of chemotherapy, and four (33\%) had received previous immunotherapy. No partial or complete responses were observed. At the lower dose level two patients had SD, while at the highest level five out of six patients presented SD. A longer survival of uveal melanoma patients compared to cutaneous melanoma was found. Acute toxicity involved flu-like syndrome with chills, fever, headache, myalgias, nausea, vomiting, and diarrhea $4-6 \mathrm{~h}$ after infusion for 2-4 days. This phase I study shows IV administration of adenovirus ICOVIR-5 is well tolerated [25].

\section{Targeting Inhibitory Molecules: CSF1Ri and IDOi}

\section{CSF1R Inhibitors}

Immune modulation of macrophages has been found to represent a novel approach to anticancer therapy. Colony-stimulating factor 1 receptor (CSF1R) is a transmembrane protein receptor for colony stimulating factor 1 , altering macrophage production and function, which is normally overexpressed on tumor-infiltrating macrophages [26]. Binding of CSF1 promotes the activation of the CSF1R-mediated pathway, thus inducing differentiation and macrophage survival, which are linked to worse survival outcomes. According to this principle, new molecules targeting CSF1 (pexidartinib, PLX7486, ARRY-382, JNJ-40346527, BLZ945, emactuzumab, AMG820, IMC-CS4) have been developed and entered early phase clinical trials 
for the treatment of solid tumors, including melanoma [27].

\section{IDO Inhibitors}

Indoleamine 2,3-dioxygenase 1 (IDO1), which regulates tryptophan catabolism, plays a pivotal role in the cancer immune surveillance mechanism. It is associated with the progression of many types of tumors, including melanoma [28]. However, there are few data regarding IDO modulation of tumor cells and the effect of BRAF inhibitor (BRAFi) treatment and resistance. Although several clinical trials using IDO inhibitor as monotherapy have already been conducted, not reporting promising results, combinations of the IDOi epacadostat with pembrolizumab or nivolumab showed response rates in untreated melanoma cohorts of approximately 60\% [29]. In 2019, Long et al. [30] conducted a randomized, placebo-controlled, double-blind phase 3 trial of pembrolizumab with or without epacadostat for patients with stage III or IV melanoma (ECHO301/KEYNOTE-252). The combination treatment did not improve PFS or OS compared with placebo plus pembrolizumab, and the trial was terminated prematurely.

\section{T Cell Agonists}

$\mathrm{T}$ cell agonists have been identified as new targets for anti-cancer therapy, acting through the upregulation of cytotoxic $\mathrm{T}$ cell activity and having a key role in the activation of regulatory $\mathrm{T}$ cells. These targets, including OX40, GITR, $41 \mathrm{BB}$, and $\mathrm{CD} 27$, are co-stimulatory receptors expressed on CD4 and CD8 cells that act by activating nuclear factor kappa $\mathrm{B}$ and c-Jun-Nterminal kinase signaling cascade signaling transcription factors thus facilitating CD4 and CD8 $\mathrm{T}$ cell differentiation.

\section{OX40}

The antitumor activity of OX40 antibody is associated with the infiltration of $\mathrm{T}$ cells and the proliferation of effector $\mathrm{T}$ cells at tumor sites. Currently, there are several clinical trials evaluating the role of anti-OX40 agents in patients with melanoma and other solid tumors. The phase I study evaluating PF-04518600, a novel fully human IgG2 agonistic $\mathrm{mAb}$ against human OX40 in patients with advanced solid tumors, led to anti-tumor responses and was well tolerated [31]. Correspondingly, the most recent first-in-human study evaluating the agonistic antibody MEDI0562 also showed good tolerability with the majority of AEs being of grade 1 or 2 [31].

\section{GITR}

Glucocorticoid-induced tumor necrosis factor receptor family-related protein (GITR) is expressed in both effector and regulatory T cells. Several trials have studied the efficacy and safety profile of anti-GITR monoclonal antibodies in patients with advanced melanoma. In 2019, a phase I clinical trial evaluating the fully humanized GITR monoclonal antibody TRX518 (NCT01239134) reported no substantial clinical responses with tumor progression observed in 29 patients during treatment. Similar results were also seen in another phase I study that evaluated the GITR monoclonal antibody $(\mathrm{mAb})$ AMG228. To date, other trials are currently assessing the efficacy of the $\mathrm{mAb}$ INGAGN1876, and results are not yet available [32].

\section{4-1BB}

4-1BB, also known as CD137 and TNFSFR9, is a stimulatory coreceptor expressed on $\mathrm{T}, \mathrm{NK}$, and dendritic cells; binding to its corresponding ligand, it causes proliferation and survival of effector T cells and cytokine release. Urelumab (BMS-663513, clone 10C7) and utomilumab (PF-05082566) are two CD137/4-1BB agonistic mAbs under clinical evaluation. In a phase I trial of utomilumab including melanoma and non-melanoma patients, clinical objective responses in tumor regression were reported. Nevertheless, treatment-related adverse events, especially immune-related toxicities, were also reported; further preclinical studies demonstrated utomilumab to be better tolerated than urelumab [33, 34]. Currently, there are ongoing studies of CD137 agonists for the treatment in patients with late-stage melanoma. 


\section{CD27}

CD27 (also known as TNFRSF7) is mainly expressed on thymocytes, naive $\mathrm{T}$ cells, memory $\mathrm{T}$ cells, and a subset of NK cells. CD27 engagement with its ligand CD70 delivers a co-stimulatory signal to further promote the differentiation and expansion of $\mathrm{T}$ cells and their activation. In a phase I trial evaluating the use of varilumab, a novel agonist anti-CD27 $\mathrm{mAb}$, in melanoma patients, modest clinical responses were achieved [35]. Further investigations in larger cohorts of patients are still needed.

\section{TLR}

Toll-like receptors (TLRs) are mammalian homologs of Drosophila Toll protein, and ten have been identified in humans. TLRs of the cells of the innate immune system recognize pathogen-associated molecular patterns and activate the immune system through the activation of nuclear factor-KB and interferon regulatory factor signaling pathways. In the last decade TLR agonists have been studied for their anti-tumoral activity. In 2018, a phase I trial demonstrated evidence of efficacy in patients with PD-1 refractory metastatic melanoma. Three patients were treated with IMO-2125 (tilsotolimod), a TLR9 agonist, with one patient reporting complete remission and two reporting partial remission of their tumor [36].

\section{Adoptive T Cell Therapy}

The use of selected in vitro tumor-infiltrating lymphocytes (TIL), recognizing melanoma antigens, has also been studied to treat highly selected patients with unresectable stage IV melanoma. Initially, this treatment showed promising results with ORR ranging between 38 and 55\%, with significant durable responses. Nevertheless, there are several limitations regarding this treatment, such as being expensive and time consuming because of the selection of specific $T$ cells acting against melanoma and the attrition rate noted during TIL generation as a result of tumor progression. The combination of checkpoint inhibition with TIL to better control tumor progression during adoptive $\mathrm{T}$ cell transfer is under investigation.

\section{Cytokines}

\section{L19IL2 (Darleukin)}

Engineered cytokine products represent promising agents for the treatment of immunogenic tumors, such as malignant melanoma, in addition to immune checkpoint inhibitors. L19IL2 is a fully human fusion protein consisting of the L19 antibody specific to the alternatively spliced extra-domain $\mathrm{B}$ of fibronectin fused to human interleukin-2 [38]. A controlled, randomized phase II clinical trial assessed its potential in treating advanced metastatic melanoma. A group of patients enrolled received dacarbazine as a single agent, while two other groups received L19IL2 in two different schedules. Sixty-nine patients with stage IV melanoma were enrolled (24 in the dacarbazine arm, 23 and 22 in the other combination arms, respectively), and statistically significant benefit in terms of ORR was reported in patients receiving L19IL2 in combination with dacarbazine compared to dacarbazine as single agent [39].

\section{Bempegaldesleukin (BEMPEG; NKTR-214)}

Bempegaldesleukin is a first-in-class CD122preferential IL-2 pathway agonist, designed to act through the IL- $2 \beta \gamma$ receptor and to selectively stimulate an antitumor response. A recent phase II randomized trial PIVOT-02 trial evaluating the combination treatment of bempegaldesleukin plus nivolumab as first-line therapy in metastatic melanoma showed positive results in term of efficacy and tolerability. A phase III, global randomized trial (PIVOT IO 001) comparing bempegaldesleukin plus nivolumab with nivolumab alone in patients with previously untreated, unresectable or metastatic melanoma is still going on, and the results have not been published yet [40].

Daromun Daromun is an intralesional immunocytokine obtained from the fusion of recombinant human proteins (cytokines IL-2 and TNF- $\alpha$ ) to the monoclonal L19 antibody. 
Although early clinical studies have already demonstrated its efficacy in local tumor destruction and in treating metastatic disease, the phase III Neo-DREAM study, is currently investigating the efficacy of neoadjuvant Daromun for resectable stage IIIB/c melanoma [41].

\section{Bevacizumab}

Systemic anti-vascular endothelial growth factor (VEGF) bevacizumab (Avastin, Genentech, San Francisco, CA, USA) has been studied in combination with other therapeutic agents for treating metastatic melanoma. An open-label randomized phase II trial characterized the efficacy and safety of bevacizumab in combination with carboplatin plus paclitaxel (CPB) in 114 patients with advanced melanoma. Results were significantly better in patients receiving bevacizumab in addition to CPB than in those who received CPB alone [42]. A phase III study should be performed to confirm these results (ClinicalTrials.gov identifier: NCT02023710).

\section{Combination Treatment}

Acquired resistance to both targeted therapy and immunotherapy has been frequently described, and, to avoid this problem, different combination therapies have been the objective of several studies. Numerous combinations including pairs and triplets of drugs with the highest clinical efficacy and minimum adverse events have been tested in unresponsive patients with advanced melanoma, especially for patients with BRAF V600e mutant disease, which constitutes $50 \%$ of advanced melanoma. Below, completed phase III trials of FDA-approved combination therapies in advanced melanoma will be reported.

\section{Immunotherapy Combination}

The immunotherapy combination of nivolumab (a programmed death [PD]-1 inhibitor) plus ipilimumab (a cytotoxic T-lymphocyte antigen-4 [CTLA-4] inhibitor) has been FDAapproved as first-line treatment for patients with BRAF-mutant melanoma. In the phase III CheckMate 067 study (NCT01844505), involving patients with stage III/IV melanoma, a group was treated with ipilimumab plus nivolumab (4 doses at $3 \mathrm{mg} / \mathrm{kg}+3 \mathrm{mg} / \mathrm{kg}$ every 2 weeks) and the other group with ipilimumab alone [43]. At 5-year follow-up the group of patients treated with the combination therapy reported an overall survival of $60 \%$ [8].

\section{Targeted Therapy Combination}

The introduction of BRAF inhibitors for patients with BRAF V600-mutated metastatic melanoma has enlarged the armamentarium of treatment for clinicians; however, resistance to BRAF inhibitors has been described. Combining BRAF and MEK inhibition has represented a new strategy for better inhibition of the MAPK pathway compared to BRAF inhibition alone. The association of cobimetinib (a MEK inhibitor) and vemurafenib (a BRAF inhibitor) has been FDA approved as standard treatment for patients with $\mathrm{BRAF}^{\mathrm{V} 600}$-mutated melanoma. Clinical data pooled from the BRIM-2, BRIM-3, BRIM-7, and coBRIM studies, involving patients treated with vemurafenib or cobimetinib plus vemurafenib, have demonstrated the combination therapy to be more effective in terms of tumor reduction and progression-free survival, supporting the combination BRAF and MEK inhibition as a standard of care in this disease setting [44]. Moreover, the association of the BRAF inhibitor dabrafenib (at a dose of $150 \mathrm{mg}$ twice daily) plus the MEK inhibitor trametinib ( $2 \mathrm{mg}$ once daily) studied in the COMBI-d and COMBI-v trials also showed excellent results leading to long-term benefit in approximately one third of the patients who had unresectable or metastatic melanoma with BRAF V600E or V600K mutation. In particular, a total of 563 patients were randomly assigned to receive dabrafenib plus trametinib (211 in the COMBI-d trial and 352 in the COMBI-v trial), reporting PFS rates of $21 \%$ (95\% confidence interval [CI], 17-24) at 4 years and 19\% (95\% CI, 15-22) at 5 years. The OS rates were $37 \%$ (95\% CI, 33-42) at 4 years and 34\% (95\% CI, $30-38)$ at 5 years. A complete response occurred in 109 patients (19\%) and was associated with an improved long-term outcome, with an OS rate of $71 \%(95 \% \mathrm{CI}, 62-79)$ at 5 years [45]. Another FDA-approved BRAF/MEK inhibitor 
combination is encorafenib and binimetinib ( $450 \mathrm{mg}$ once daily $+45 \mathrm{mg}$ twice daily). Phase III data of 577 patients randomized to encorafenib plus binimetinib, encorafenib alone, or vemurafenib alone showed an ORR of $63 \%$, $51 \%$, and $40 \%$ and PFS of $14.9,9.6$, and 7.3 months, respectively. Interestingly, grade 3 to 4 AEs occurred less frequently in the combination group (58\%) compared to encorafenib alone (66\%) and vemurafenib alone (63\%) [46].

\section{CONCLUSIONS}

In the last decade clinical research on melanoma has completely changed the scenario of therapeutic approaches for patients with unresectable advanced melanoma. Recent publications have reported outstanding results in the adjuvant treatment of stage IIIA to fully resected stage IV melanoma. Multiple targets for drug development have been identified and are currently undergoing development. Targeting immunological pathways (CTLA4, PD1, TNF, and TNFR superfamilies, immunoglobulin superfamily, B7, CD28, TLR-9, TLR-3, NK cells, and other mediators like IDO, TGF $\beta$, CXCR4, adenosine pathway, VEGF), driver mutations (BRAF, NRAS, ckit and corresponding signaling pathways), and genetically engineered viruses and vaccines have been shown to be valid treatment options used alone or in combination to avoid resistance [5]. Nivolumab remains an efficacious adjuvant treatment for patients with resected high-risk melanoma, with a safety profile that is more tolerable than that of ipilimumab [9]. Moreover, whether all patients at stage III or IV should receive adjuvant therapy and how to treat patients at stage IIB, IIC, or IIIA in adjuvant therapy remain controversial issues. In the last years, targeted nanoformulations have been explored for their potential in melanoma treatment, and promising results have been obtained [47-49]. Finally, regarding the decision-making on the appropriate adjuvant therapy for patients with resected stage III or IV melanoma, not only the efficacy but also the toxicity profile and medical history of the patient should be taken into account. Further reports of ongoing trials and research are needed to improve the efficacy of neoadjuvant and adjuvant therapy and to determine the standard adjuvant therapy for patients with high-risk advanced melanoma.

\section{ACKNOWLEDGEMENTS}

Funding. No funding or sponsorship was received for this study or publication of this article.

Authorship. All named authors meet the International Committee of Medical Journal Editors (ICMJE) criteria for authorship for this article, take responsibility for the integrity of the work as a whole, and have given their approval for this version to be published.

Author Contributions. Conception and design: Alessia Villani, Sonia Ocampo, Massimiliano Scalvenzi. Acquisition of data, analysis, and interpretation of data: Alessia Villani, Sonia Ocampo. Drafting the article: Alessia Villani, Sonia Ocampo. Critical revisión: Alessia Villani, Sonia Ocampo, Massimiliano Scalvenzi, Jorge Ocampo, Gabriella Fabbrocini. Final approval of the version to be published: Alessia Villani, Sonia Ocampo, Massimiliano Scalvenzi, Jorge Ocampo, Gabriella Fabbrocini.

Disclosures. Alessia Villani, Massimiliano Scalvenzi, Gabriella Fabbrocini, Jorge OcampoCandiani, and Sonia Sofía Ocampo-Garza have nothing to disclose.

Compliance with Ethics Guidelines. This article is based on previously conducted studies and does not contain any studies with human participants or animals performed by any of the authors.

Data Availability. Data are available in the manuscript; tables are included in the article.

Open Access. This article is licensed under a Creative Commons Attribution-NonCommercial 4.0 International License, which permits any non-commercial use, sharing, adaptation, 
distribution and reproduction in any medium or format, as long as you give appropriate credit to the original author(s) and the source, provide a link to the Creative Commons licence, and indicate if changes were made. The images or other third party material in this article are included in the article's Creative Commons licence, unless indicated otherwise in a credit line to the material. If material is not included in the article's Creative Commons licence and your intended use is not permitted by statutory regulation or exceeds the permitted use, you will need to obtain permission directly from the copyright holder. To view a copy of this licence, visit http://creativecommons.org/licenses/bync/4.0/.

\section{REFERENCES}

1. Testori AAE, Ribero S, Indini A, Mandalà M. Adjuvant treatment of melanoma: recent developments and future perspectives. Am J Clin Dermatol. 2019;20:817-27. https://doi.org/10.1007/s40257019-00456-4.

2. Wada-Ohno M, Ito T, Furue M. Adjuvant therapy for melanoma. Curr Treat Options Oncol. 2019;20: 63.

3. Nguyen K, Hignett E, Khachemoune A. Current and emerging treatment options for metastatic melanoma: a focused review. Dermatol Online J. 2020;26:13030/qt24g3k7z5.

4. Bolognia JL, Schaffer JV, Cerroni L. Dermatology. Fourth Ed. 2017. p. 2008.

5. Luther C, Swami U, Zhang J, Milhem M, Zakharia Y. Advanced stage melanoma therapies: detailing the present and exploring the future. Crit Rev Oncol Hematol. 2019;133:99-111. https://doi.org/10. 1016/j.critrevonc.2018.11.002.

6. Mason R, Au L, Ingles Garces A, Larkin J. Current and emerging systemic therapies for cutaneous metastatic melanoma. Expert Opin Pharmacother. 2019;20:1135-52. 14656566.2019.1601700.

7. Warner AB, Palmer JS, Shoushtari AN, Goldman DA, Panageas KS, Hayes SA, et al. Long-term outcomes and responses to retreatment in patients with melanoma treated with PD-1 blockade. J Clin Oncol. 2020;38:1655-63.
8. Larkin J, Minor D, D'Angelo S, Neyns B, Smylie M, Miller WH, et al. Overall survival in patients with advanced melanoma who received nivolumab versus investigator's choice chemotherapy in CheckMate 037: a randomized, controlled, open-label phase III trial. J Clin Oncol. 2018;36:383-90.

9. Weber J, Mandala M, Del Vecchio M, Gogas HJ, Arance AM, Cowey CL, et al. Adjuvant nivolumab versus ipilimumab in resected stage Iii or IV melanoma. N Engl J Med. 2017;377:1824-35.

10. Ascierto PA, Del Vecchio M, Mandalá M, Gogas H, Arance AM, Dalle $S$, et al. Adjuvant nivolumab versus ipilimumab in resected stage IIIB-C and stage IV melanoma (CheckMate 238): 4-year results from a multicentre, double-blind, randomised, controlled, phase 3 trial. Lancet Oncol. 2020;21: 1465-77.

11. Hamid O, Robert C, Daud A, Hodi FS, Hwu WJ, Kefford R, et al. Five-year survival outcomes for patients with advanced melanoma treated with pembrolizumab in KEYNOTE-001. Ann Oncol. 2019;30:582-8.

12. Kang SP, Gergich K, Lubiniecki GM, De ADP, Chen C, Melissa AB. Pembrolizumab KEYNOTE-001: an adaptive study leading to accelerated approval for two indications and a companion diagnostic. Ann Oncol. 2017;28:1388-98.

13. Eggermont AMM, Blank CU, Mandala M, Long GV, Atkinson V, Dalle S, et al. Adjuvant pembrolizumab versus placebo in resected stage III melanoma. N Engl J Med. 2018;378:1789-801.

14. Chanal J, Kramkimel N, Ratour C, Aractingi S, Guégan S, Avril MF. Pembrolizumab for unresectable or metastatic melanoma in patients older than 85 years of age. Dermatology. 2019;235: 219-24.

15. Drysdale E, Peng Y, Nguyen P, Baetz T, Hanna TP. A population-based study of the treatment effect of first-line ipilimumab for metastatic or unresectable melanoma. Melanoma Res. 2019;29: 635-42.

16. Lang BM, Peveling-Oberhag A, Faidt D, Hötker AM, Weyer-Elberich V, Grabbe S, et al. Long-term survival with modern therapeutic agents against metastatic melanoma-vemurafenib and ipilimumab in a daily life setting. Med Oncol. 2018;35: 24. https://doi.org/10.1007/s12032-018-1084-9.

17. Maio M, Lewis $\mathrm{K}$, Demidov L, Mandalà M, Bondarenko I, Ascierto PA, et al. Adjuvant vemurafenib in resected, BRAFV600 mutation-positive melanoma (BRIM8): a randomised, double-blind, placebo-controlled, multicentre, phase 3 trial. Lancet Oncol. 2018;19:510-20. 
18. Perez MC, Miura JT, Naqvi SMH, Kim Y, Holstein A, Lee D, et al. Talimogene laherparepvec (TVEC) for the treatment of advanced melanoma: a single-institution experience. Ann Surg Oncol. 2018;25: 3960-5. https://doi.org/10.1245/s10434-018-68030 .

19. Burke EE, Zager JS. Pharmacokinetic drug evaluation of talimogene laherparepvec for the treatment of advanced melanoma. Expert Opin Drug Metab Toxicol. 2018;14:469-73. https://doi.org/10.1080/ 17425255.2018.1455825.

20. Andtbacka RHI, Collichio F, Harrington KJ, Middleton MR, Downey G, Katarina Ö, et al. Final analyses of OPTiM: a randomized phase III trial of talimogene laherparepvec versus granulocyte-macrophage colony-stimulating factor in unresectable stage III-IV melanoma. J Immunother Cancer. 2019;7:145.

21. Mohr P, Haferkamp S, Pinter A, Weishaupt C, Huber MA, Downey G, et al. Real-world use of talimogene laherparepvec in german patients with stage IIIB to IVM1a melanoma: a retrospective chart review and physician survey. Adv Ther. 2019;36:101-17.

22. Franke V, Berger DMS, Klop WMC, van der Hiel B, van de Wiel BA, ter Meulen $S$, et al. High response rates for T-VEC in early metastatic melanoma (stage IIIB/C-IVM1a). Int J Cancer. 2019;145:974-8.

23. Zhou AY, Wang DY, McKee S, Ye F, Wen CC, Wallace DE, et al. Correlates of response and outcomes with talimogene laherperpvec. J Surg Oncol. 2019;120:558-64. https://doi.org/10.1002/jso. 25601.

24. Louie RJ, Perez MC, Jajja MR, Sun J, Collichio F, Delman KA, et al. Real-world outcomes of talimogene laherparepvec therapy: a multi-institutional experience. J Am Coll Surg. 2019;228:644-9. https://doi.org/10.1016/j.jamcollsurg.2018.12.027.

25. García M, Moreno R, Gil-Martin M, Cascallò M, De Olza MO, Cuadra C, et al. A phase 1 trial of oncolytic adenovirus ICOVIR-5 administered intravenously to cutaneous and uveal melanoma patients. Hum Gene Ther. 2019;30:352-64.

26. Ambrosi L, Khan S, Carvajal RD, Yang J. Novel targets for the treatment of melanoma. Curr Oncol Rep. 2019;21:97. https://doi.org/10.1007/s11912019-0849-4.

27. Osipov A, Saung MT, Zheng L, Murphy AG. Small molecule immunomodulation: the tumor microenvironment and overcoming immune escape. J Immunother Cancer. 2019;7:224. https:// doi.org/10.1186/s40425-019-0667-0.
28. Mellor AL, Keskin DB, Johnson T, Chandler $\mathrm{P}$, Munn DH. Cells expressing indoleamine 2,3 dioxygenase inhibit Tcell responses. J Immunol. 2002;168:3771-6.

29. Mitchell TC, Hamid O, Smith DC, et al. Epacadostat plus pembrolizumab in patients with advanced solid tumors: phase I results from a multicenter, open-label phase I/II trial (ECHO-202/KEYNOTE037). J Clin Oncol. 2018;36:3223-30. https://doi. org/10.1200/JCO.2018.78.9602.

30. Long GV, Dummer R, Hamid O, Gajewski TF, Caglevic C, Dalle S, Arance A, Carlino MS, Grob JJ, Kim TM, Demidov L, Robert C, Larkin J, Anderson JR, Maleski J, Jones M, Diede SJ, Mitchell TC. Epacadostat plus pembrolizumab versus placebo plus pembrolizumab in patients with unresectable or metastatic melanoma (ECHO-301/KEYNOTE-252): a phase 3, randomised, double-blind study. Lancet Oncol. 2019;20:1083-97. https://doi. org/10.1016/S1470-2045(19)30274-8 (Epub 2019 Jun 17).

31. Glisson BS, Leidner RS, Ferris RL, Powderly J, Rizvi NA, Keam B, Schneider R, Goel S, Ohr JP, Burton J, Zheng Y, Eck S, Gribbin M, Streicher K, Townsley DM, Patel SP. Safety and clinical activity of MEDI0562, a humanized OX40 agonist monoclonal antibody, in adult patients with advanced solid tumors. Clin Cancer Res. 2020;26:5358-67. https:// doi.org/10.1158/1078-0432.CCR-19-3070 (Epub 2020 Aug 14).

32. Zappasodi R, Sirard C, Li Y, Budhu S, Abu-Akeel M, Liu C, Yang X, Zhong H, Newman W, Qi J, Wong P, Schaer D, Koon H, Velcheti V, Hellmann MD, Postow MA, Callahan MK, Wolchok JD, Merghoub T. Rational design of anti-GITR-based combination immunotherapy. Nat Med. 2019;25:759-66. https://doi.org/10.1038/s41591-019-0420-8 (Epub 2019 Apr 29).

33. Segal NH, He AR, Doi T, Levy R, Bhatia S, Pishvaian MJ, Cesari R, Chen Y, Davis CB, Huang B, Thall AD, Gopal AK. Phase I study of single-agent utomilumab (PF-05082566), a 4-1BB/CD137 agonist, in patients with advanced cancer. Clin Cancer Res. 2018;24:1816-23. https://doi.org/10.1158/10780432.CCR-17-1922.

34. Segal NH, Logan TF, Hodi FS, McDermott D, Melero I, Hamid O, Schmidt H, Robert C, Chiarion-Sileni V, Ascierto PA, Maio M, Urba WJ, Gangadhar TC, Suryawanshi S, Neely J, Jure-Kunkel M, Krishnan S, Kohrt H, Sznol M, Levy R. Results from an integrated safety analysis of urelumab, an agonist antiCD137 monoclonal antibody. Clin Cancer Res. 2017;23:1929-36. https://doi.org/10.1158/10780432.CCR-16-1272. 
35. Burris HA, Infante JR, Ansell SM, Nemunaitis JJ, Weiss GR, Villalobos VM, Sikic BI, Taylor MH, Northfelt DW, Carson WE 3rd, Hawthorne TR, Davis TA, Yellin MJ, Keler T, Bullock T. Safety and activity of varlilumab, a novel and first-in-class agonist anti-CD27 antibody, in patients with advanced solid tumors. J Clin Oncol. 2017;35: 2028-36. https://doi.org/10.1200/JCO.2016.70. 1508.

36. Wang D, Jiang W, Zhu F, Mao X, Agrawal S. Modulation of the tumor microenvironment by intratumoral administration of IMO-2125, a novel TLR9 agonist, for cancer immunotherapy. Int J Oncol. 2018;53:1193-203. https://doi.org/10.3892/ ijo.2018.4456.

37. Mullinax JE, Hall M, Prabhakaran S, et al. Combination of ipilimumab and adoptive cell therapy with tumor-infiltrating lymphocytes for patients with metastatic melanoma. Front Oncol. 2018;8:44.

38. Penichet ML, Dela Cruz JS, Shin SU, Morrison SL. A recombinant IgG3-(IL-2) fusion protein for the treatment of human HER2/neu expressing tumors. Hum Antibodies. 2001;10:43-9.

39. Weide B, Eigentler T, Catania C, Ascierto PA, Cascinu S, Becker JC, Hauschild A, Romanini A, Danielli R, Dummer R, Trefzer U, Elia G, Neri D, Garbe C. A phase II study of the L19IL2 immunocytokine in combination with dacarbazine in advanced metastatic melanoma patients. Cancer Immunol Immunother. 2019;68:1547-59. https:// doi.org/10.1007/s00262-019-02383-z.

40. Khushalani NI, Diab A, Ascierto PA, Larkin J, Sandhu S, Sznol M, Koon HB, Jarkowski A, Zhou M, Statkevich P, Geese WJ, Long GV. Bempegaldesleukin plus nivolumab in untreated, unresectable or metastatic melanoma: phase III PIVOT IO 001 study design. Future Oncol. 2020;16: 2165-75. https://doi.org/10.2217/fon-2020-0351.

41. Miura JT, Zager JS. Neo-DREAM study investigating Daromun for the treatment of clinical stage IIIB/C melanoma. Future Oncol. 2019;15:3665-74. https://doi.org/10.2217/fon-2019-0433.

42. Yan X, Sheng X, Chi Z, Si L, Cui C, Kong Y, Tang B, Mao L, Wang X, Lian B, Li S, Bai X, Zhou L, Dai J, Yao H, Guo J. Randomized phase II study of bevacizumab in combination with carboplatin plus paclitaxel in patients with previously untreated advanced mucosal melanoma. J Clin Oncol. 2021;2021:JCO200902. https://doi.org/10.1200/ JCO.20.00902.
43. Larkin J, Hodi FS, Wolchok JD. Combined nivolumab and ipilimumab or monotherapy in untreated melanoma. N Engl J Med. 2015;373:1270-1. https:// doi.org/10.1056/NEJMc1509660.

44. Tarhini AA, Toor K, Chan K, McDermott DF, Mohr P, Larkin J, Hodi FS, Lee CH, Rizzo JI, Johnson H, Moshyk A, Rao S, Kotapati S, Atkins MB. A matching-adjusted indirect comparison of combination nivolumab plus ipilimumab with BRAF plus MEK inhibitors for the treatment of BRAF-mutant advanced melanoma ${ }^{\text {th }}$. ESMO Open. 2021;6: 100050. https://doi.org/10.1016/j.esmoop.2021. 100050.

45. Robert C, Grob JJ, Stroyakovskiy D, Karaszewska B, Hauschild A, Levchenko E, Chiarion Sileni V, Schachter J, Garbe C, Bondarenko I, Gogas H, Mandalá M, Haanen JBAG, Lebbé C, Mackiewicz A, Rutkowski P, Nathan PD, Ribas A, Davies MA, Flaherty KT, Burgess P, Tan M, Gasal E, Voi M, Schadendorf D, Long GV. Five-year outcomes with dabrafenib plus trametinib in metastatic melanoma. N Engl J Med. 2019;381:626-36. https://doi. org/10.1056/NEJMoa1904059.

46. Ascierto PA, Dummer R, Gogas HJ, Flaherty KT, Arance A, Mandala M, Liszkay G, Garbe C, Schadendorf D, Krajsova I, Gutzmer R, de Groot JWB, Loquai C, Gollerkeri A, Pickard MD, Robert C. Update on tolerability and overall survival in COLUMBUS: landmark analysis of a randomised phase 3 trial of encorafenib plus binimetinib vs vemurafenib or encorafenib in patients with BRAF V600-mutant melanoma. Eur J Cancer. 2020;126: 33-44. https://doi.org/10.1016/j.ejca.2019.11.016.

47. Robert C, Grob JJ, Stroyakovskiy D, et al. Five-year outcomes with dabrafenib plus trametinib in metastatic melanoma. N Engl J Med. 2019;381(7): 626-36. https://doi.org/10.1056/NEJMoa1904059.

48. Villani A, Fabbrocini G, Costa C, Scalvenzi M. Melanoma screening days during the coronavirus disease 2019 (COVID-19) pandemic: strategies to adopt. Dermatol Ther (Heidelb). 2020;10(4):525-7. https://doi.org/10.1007/s13555-020-00402-X (Epub 2020 May 28).

49. Eggermont AMM, Dummer R. The 2017 complete overhaul of adjuvant therapies for high-risk melanoma and its consequences for staging and management of melanoma patients. Eur J Cancer. 2017;86:101-5. 